\title{
Enhancing the photoluminescence of surface anchored metal- organic frameworks: mixed linkers and efficient acceptors
}

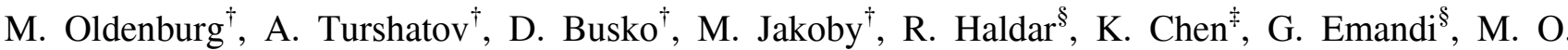 \\ Senge $^{\S}$, C. Wöll $\|$, J.M. Hodgkiss ${ }^{\ddagger}$, B. S. Richards ${ }^{\dagger, \perp}$, I. A. Howard ${ }^{\dagger, \perp, *}$ \\ ${ }^{\dagger}$ Institute of Microstructure Technology, Karlsruhe Institute of Technology, Hermann-von-Helmholtz-Platz 1, 76344 Eggen- \\ stein-Leopoldshafen, Germany \\ ${ }^{\ddagger}$ The MacDiarmid Institute for Advanced Materials and Nanotechnology, and School of Chemical and Physical Sciences, \\ Victoria University of Wellington, Wellington 6140, New Zealand \\ ${ }^{\S}$ School of Chemistry, SFI Tetrapyrrole Laboratory, Trinity Biomedical Science Institute, 152-160 Pearse Street, Trinity \\ College Dublin, The University of Dublin, Dublin 2, Ireland \\ "Institute of Functional Interfaces, Karlsruhe Institute of Technology, Hermann-von-Helmholtz-Platz 1, 76344 Eggenstein- \\ Leopoldshafen, Germany \\ ${ }^{\perp}$ Light Technology Institute, Karlsruhe Institute of Technology, Engesserstrasse 13, 76131 Karlsruhe, Germany \\ ian.howard@kit.edu, +49 721 608-28398
}

\begin{abstract}
We present two approaches to enhance the photoluminescence quantum yield (PLQY) of surface-anchored metalorganic frameworks (SURMOFs). In the first approach we fabricate SURMOFs from a mix of an emissive linker with an opticallyinert linker of equivalent length, diluting the emissive linker while maintaining the SURMOF structure. This approach enhances the internal PLQY. However, the increase in internal PLQY is achieved at the expense of a drastic reduction in optical absorption, thus the external PLQY remains low. To overcome this limitation, a second approach is explored wherein energy-accepting guest chromophores are infiltrated into the framework of the active linker. At the correct acceptor concentration, an internal PLQY of 52\% three times higher than the previous approach - is achieved. Additionally, the absorption remains strong leading to an external PLQY of $8 \%$, an order of magnitude better than the previous approach. Using this strategy, we demonstrate that SURMOFs can achieve PLQYs similar to their precursor chromophores in solution. This is of relevance to SURMOFs as emitter layers in general, and we examine the optimized emitter layer as part of a photon upconversion (UC) SURMOF heterostructure. Surprisingly, the same PLQY is not observed after triplet-triplet annihilation the UC heterostructure as after its normal photoexcitation (although the UC layers exhibit low thresholds consistent with those reported in our previous work). We discuss the potential bottlenecks in energy transport that could lead to this unexpected reduction in PLQY after excitation via triplet-triplet annihilation, and how future design of SURMOF UC multilayers could overcome these limitations.
\end{abstract}

\section{Introduction}

Metal-organic frameworks (MOFs) are prepared by coordination of organic linkers to metal centers, yielding porous, crystalline frameworks. ${ }^{1,2}$ The well-defined, highly-ordered arrangement of the building blocks make MOFs interesting model systems for observing energy transfer between chromophore linkers, ${ }^{3-10}$ as well as between linker and guest molecules that are loaded into the MOF. ${ }^{11,12}$ Due to the high local order, long exciton diffusion lengths are observed in MOFs. ${ }^{8,11,13,14}$ Appealing energy transport properties within MOFs support interest in developing their properties towards device applications, ${ }^{15,16}$ these applications include as semiconductors in photovoltaic ${ }^{17-19}$ and thermoelectric ${ }^{20}$ devices, but also potentially as metallic-like conductors, despite their porous nature. ${ }^{21,22}$ Furthermore, layer-by-layer fabrication strategies allow for the facile construction of multilayer heterostructures that expand the potential application space of MOFs. ${ }^{23,24}$ We have previously demonstrated triplet exciton transport across the heterojunction of a SURMOF heterostructure designed for photon upconversion (UC) via triplet-triplet annihilation (TTA-UC) ${ }^{25}$ We reported low thresholds $\left(1 \mathrm{~mW} / \mathrm{cm}^{2}\right)$, but the quantum efficiency of the upconversion was too low to measure, which was ascribed to a very limited photoluminescent quantum yield (PLQY) in the emitter layer. Other papers claiming significantly lower thresholds and higher UC PLQYs in MOF crystals have recently been retracted. In order to carefully examine these open issues - and to advance the use of MOFs for light-emission applications - we pursue two strategies for increasing PLQY of SURMOFs. This is of relevance for optimizing UC in these crystalline systems, but also more generally for the pursuit of device applications.

Currently, there is significant progress towards the application of MOFs for illumination applications. ${ }^{26}$ However, the PLQY of linker chromophores often drops when they are arranged in close proximity within the crystalline lattice of a MOF. For example, the PLQY of MOFs recently used in a white light emitting diode was only a modest $10 \%{ }^{26}$ Strategies to improve the PLQY of MOFs are of significant interest for their deployment in a variety of applications, ranging from energy harvesting to illumination and the afore-mentioned photon UC.

In this work, we investigate two strategies for the enhancement of PLQY of thin film SURMOFs. The first strategy involves constructing SURMOFs that contain a controlled mixture of optically-active (chromophore) and opticallyinactive (spacer) linkers. As the length of the chromophore and spacer linkers are the same, the chromophore linkers can be 'diluted' while the structure of the SURMOF remains unchanged. By thus diluting the optically-active chromophore 
linkers we could increase the PLQY, demonstrating that the interaction of neighboring chromophore molecules in the SURMOF reduces their PLQY. However, the optical absorption of the thin films is also reduced by this dilution; the optical density of the films becomes very small when the active chromophores are sparse. When employed as a phosphor layer in lighting, strong absorption is required, whereas the application in photon UC by triplet-triplet annihilation or energy harvesting requires good exciton transport. As both these properties are lost in the system of sparse active chromophores, the practical applications of this first strategy may be limited

We therefore pursued a second strategy wherein we infiltrated a SURMOF based on the optically-active chromophore linkers with an energy-accepting guest molecule that has a high PLQY. For this we chose coumarin dyes as they: 1) have high PLQYs, 2) exhibit a large overlap between their absorption spectrum and the emission spectrum of the linker chromophore, and 3) have been shown to be very effective exciton acceptors when loaded into MOFs. ${ }^{11}$ At the optimum concentration of the energy-accepting and light-emitting coumarin guest molecules, we could increase the PLQY of the coumarin@SURMOF system by an order of magnitude to values above $50 \%$. Further, the strong absorption and good exciton transport properties of the SURMOF are maintained in this approach.

In the final section of the paper we apply our techniques for improving the PLQY to the emitter layer in an UC heterostructure. We can reproduce the low thresholds reported in our previous work, ${ }^{25}$ but the UC-PLQY remains very low despite the improved PLQY of the emitter layer. We discuss this in terms of energy transport close to the heterostructure interface, and how further optimization of this transport is needed before the better PLQY of the emitter layer can be released in the upconversion heterostructure. These results confirm there is still excellent potential for the development of SURMOF heterostructures that precisely control excited-state motion in order to achieve novel and desirable macroscopic material properties.

\section{Results and Discussion}

\section{Dilution of luminescent linker ADB in TPC SURMOF}

Our first strategy to increase the PLQY was to choose two linkers of similar length, one optically active and the other passive. We used ditopic carboxylic acid linkers that result in SURMOFs with $P_{4}$ symmetry in the structural class known as SURMOF-2. ${ }^{27}$. The SURMOF fabrication was realized via spray-deposition of ethanol-based solutions under ambient conditions using the layer-by-layer approach described by Arslan et al. $^{28,29}$ As the main building block we used 4,4'(anthracene-9,10-diyl)dibenzoate (ADB). As a first attempt to increase the PLQY we diluted the ADB molecules within a SURMOF made from $p$-terphenyl-4,4'-dicarboxylate (TPC), which has a similar length as ADB but can be considered optically inert (neither absorbing nor emitting light in the wavelength range we consider.
We fabricate SURMOFs from a solution containing a mixture of $\mathrm{ADB}$ and TPC linkers. In the synthesis process, $\mathrm{Zn}^{2+}$ "paddle-wheel" metal centers are coordinated by the ditopic linkers in order to form two-dimensional sheets standing perpendicular to the substrate. ${ }^{25,28,30}$ The closest proximity between two linkers in the SURMOF structure is in the direction between sheets (Miller index (010)) and is $0.6 \mathrm{~nm}$ as extracted from X-ray diffraction data by using the Bragg condition as shown in Figure S1. However, the close distance also leads to interactions between the ADB chromophores. The resulting aggregate-like behavior limits the PLQY of the ADB-based SURMOFs. We measured a PLQY of less than $5 \%$ for a SURMOF made of solely of ADB, whereas the PLQY of the linker molecule in solution is nearly $100 \%$. We hypothesized that separating the ADB molecules from each other, as in a solution, would lead to a reduction of these losses. In order to test this hypothesis, we prepared coordination networks from mixed linker solutions. ADB was mixed during the synthesis with TPC. The strategy of using two similar or equally-sized linkers within a MOF was recently demonstrated by Dhakshinamoorthy et al. ${ }^{31}$ As TPC has the same length as ADB, it does not change the overall structure of the SURMOF. Additionally, TPC's absorption bands lie at higher energies in the ultraviolet (UV); thus in the lower energy regime relevant here TPC is optically inert.

We prepared four SURMOFs each with a different ratio between $\mathrm{ADB}$ and TPC: i) one was fabricated exclusively from $\mathrm{ADB}$; ii) one with an $\mathrm{ADB}$ to $\mathrm{TPC}$ ratio of $1: 1$; iii) one with an ADB to TPC ratio of 10:1; and iv) one consisting of neat TPC. Time-resolved PL was used to analyze the temporal evolution of the excited states in each of the SURMOFs, as shown in Figure 1a. For each measurement, the emission in the wavelength range from 350 to $600 \mathrm{~nm}$ was monitored over the time window from 0 to $10 \mathrm{~ns}$. All three samples containing ADB were excited at $350 \mathrm{~nm}$. For completeness, the SURMOF containing only TPC was excited at $350 \mathrm{~nm}$ and the absence of any measurable signal confirmed the optical inertness of this linker in the wavelength range of interest.

Figure $1 \mathrm{~b}$ demonstrates that the spectrum of the emission changes with time, indicating that two excited-state species with differing lifetimes exist within the samples. The red points indicate the center position of a Gaussian fit to the emission spectrum at various times after excitation, and the green dotted line a splined fit through these points to guide the eye. In all cases the emission shifts to the red in a similar manner with time: for pure ADB SURMOF the shift was 40 $\mathrm{nm}(0.22 \mathrm{eV})$, while the shifts for the SURMOFs containing $50 \%$ and $10 \%$ ADB were $45 \mathrm{~nm}(0.24 \mathrm{eV})$ and $50 \mathrm{~nm}(0.26$ $\mathrm{eV})$, respectively. This indicates that the nature of the two states responsible for the emission remains similar across the samples. In the following, we discuss further the nature of these two states. 
(a)

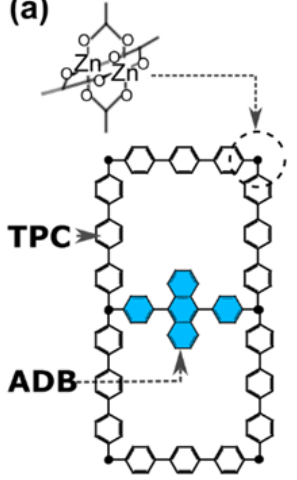

Wavelength $(\mathrm{nm})$

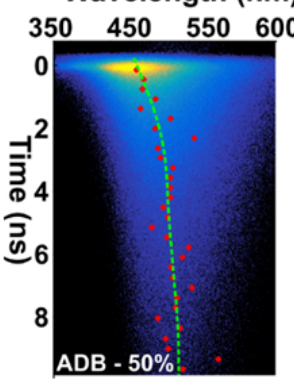

(b)
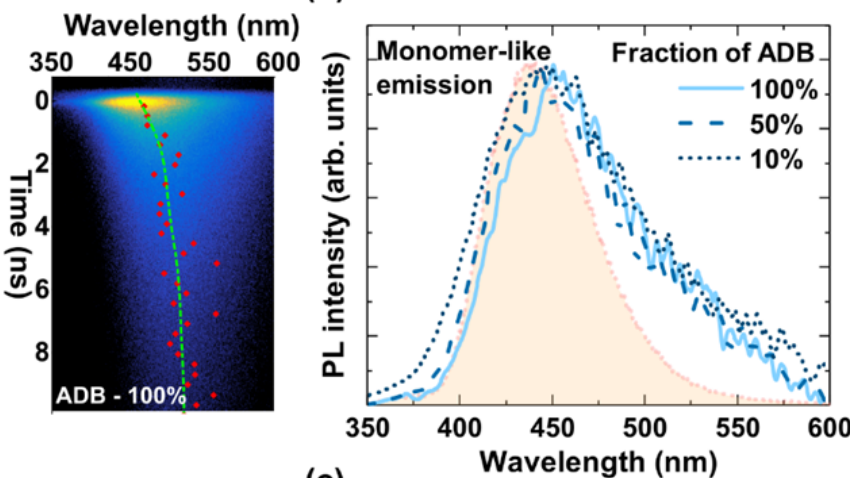

(c)

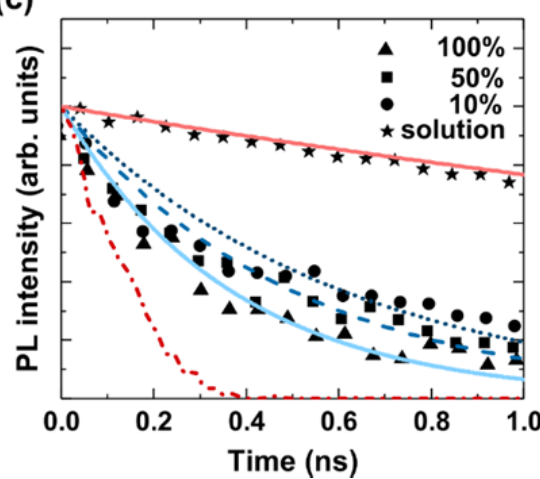

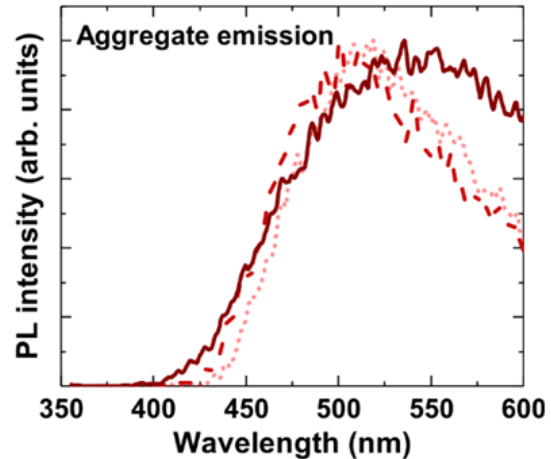

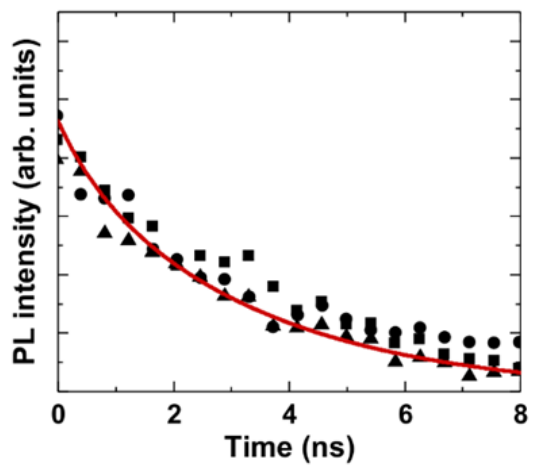

Figure 1. (a) Schematic illustration of the mixed SURMOF fabricated from a zinc center coordinated by 4,4'-(anthracene-9,10diyl)dibenzoate (ADB) and $p$-terphenyl-4,4'-dicarboxylate (TPC) linkers; and streak camera measurements of SURMOFs made from $100 \%, 50 \%$, and $10 \%$ of ADB linkers excited at $350 \mathrm{~nm}$. The red dots show the emission peak for each time and the green dashed curve is a guide to the eye provided by a spline function. The graphs in (b) and (c) show the PL spectrum and decay kinetics, respectively, of the two states responsible for the emission obtained by factoring the data shown in panel (a) using multivariate curve resolution. (b) The emission spectrum of the monomer-like (left) and aggregated state (right) for the various blend ratios. The spectrum of ADB in ethanol solution is plotted as a filled curve for comparison. (c) The population kinetics of the monomer-like and aggregate states.

In order to gain more insight into the excited-state species leading to the luminescence, we factor the time-resolved emission matrices using the method of multivariate curve resolution together with a constrained alternating least squares algorithm (MCR-ALS) using non-negativity constraints on both the spectra and kinetics. ${ }^{32}$ This gives a PL spectrum and population decay profile for each of the two species; the spectra and kinetics are shown in Figure $1 \mathrm{~b}$ and $\mathrm{c}$, respectively. The MCR-ALS reveals a short-lived component whose spectrum and kinetics are shown on the left side of panel Figure $1 \mathrm{~b}$ and 1c (in blue colors) and a long-lived component whose spectrum and kinetics are shown on the right side of the panels (in red colors). The PL spectrum for the short-lived component has an emission maximum around $445 \mathrm{~nm}$ that coincides well with the emission spectrum of ADB in solution (plotted in Figure $1 \mathrm{~b}$ as a filled curve) which has a maximum at $440 \mathrm{~nm}$. We therefore hypothesize that this blue emission derives from monomer-like states in the SURMOF (molecular excited states within the SURMOF that are similar to that of individual molecules in solution). The second component exhibits a significantly redder emission; the emission maximum for all three samples is around $510 \mathrm{~nm}$. We hypothesize that this emission arises from sites in the SURMOF where neighboring ADB chromophores can interact with each other, perhaps due to rotation of the anthracene units allowing closer contact between the anthracene cores of nearest neighbor chromophores.
In Figure 1c the lifetime profiles for the short- and longlived components are plotted. The monomer-like and the aggregated PL transients were fitted with single-exponential decay functions and a lifetime was extracted. The lifetime of the short-lived component increases as the concentration of $\mathrm{ADB}$ is reduced from $\tau_{100 \%}=0.37 \pm 0.01 \mathrm{~ns}$ to $\tau_{50 \%}=$ $0.50 \pm 0.01 \mathrm{~ns}$ to $\tau_{10 \%}=0.61 \pm 0.02 \mathrm{~ns}$. For comparison, the decay of the ADB solution is shown, along with a fit revealing its lifetime to be $4.3 \pm 0.01 \mathrm{~ns}$. These emission kinetics indicate that the lifetime of the monomer-like species is strongly quenched in the SURMOF, and more strongly quenched the higher the ADB concentration is. We will consider this further when we examine the PLQY of these films below.

In contrast, the lifetime of the long-lived state remained the same for all concentrations of ADB and is $3.3 \pm 0.3 \mathrm{~ns}$. These time-resolved data give interesting insight into the photophyiscs of the system. The amount of aggregate formation increases with increasing ADB concentration, as revealed by the decreasing lifetime of the blue "monomer-like" emission. This is consistent with more ADB chromophore linkers having at least one nearest neighbor that is also an ADB chromophore in the SURMOFs with high ADB concentrations. However, even in the $10 \%$ ADB case, many aggregates are formed. The aggregate state appears to stay the same irrespective of the concentration based on the similar lifetime and spectrum of the aggregate emission across the samples.

In Figure 2, we examine how the steady-state emission and PLQY is affected by this first strategy of changing the ADB 
linker fraction. As shown in Figure 2a, as the concentration of $\mathrm{ADB}$ is reduced the intensity of the PL increases and the maximum of the PL spectrum shifts to the blue. The maximum of the PL spectrum is at $450 \mathrm{~nm}$ for the $100 \%$ ADB sample, 440 $\mathrm{nm}$ for the $50 \%$, and $437 \mathrm{~nm}$ for the $10 \%$ sample. The blue shift with decreasing ADB fraction is consistent with more emission coming from monomer-like states in the dilute ADB samples, and less reabsorption in the dilute samples.

The PLQY was measured according to the technique presented by de Mello et al. ${ }^{33}$ We find that the average internal PLQYs were $15 \pm 2,8 \pm 4$ and $4 \pm 3 \%$ for the samples made with 10,50 , and $100 \%$ of $\mathrm{ADB}$, respectively. Note that the averages and uncertainty bounds are based on three samples made for each ratio in independent fabrication runs and that the accuracy of our PLQY setup was checked using LUMOGEN violet (BASF) for which we obtained a PLQY of $98 \%$ in good agreement with previous measurements of $99-100 \%$ from the literature. ${ }^{34}$ We further note that the internal PLQY is defined by the ratio of the number of photons emitted to the number of photons absorbed.

Given that the peak of the steady-state emission (shown in Figure 2a) closely matches the peak of the monomer-like emission found in Figure 1b, we conclude that the PLQY of the monomer-like states is much higher than the PLQY of the aggregate states and therefore the total PLQY is dominated by the emission from the monomer-like states. Under this assumption, the PLQY can be calculated as follows:

$$
\eta_{\text {SURMOF }}^{\text {int }}=\eta_{\text {solution }}^{\text {int }} \cdot \frac{\tau_{\text {monomer }}}{\tau_{\text {solution }}}
$$

where $\eta_{\text {SURMOF }}^{\text {int }}, \tau_{\text {monomer }}$ and $\eta_{\text {solution }}^{\text {int }}, \tau_{\text {solution }}$ stand for internal PLQY and PL lifetime of the monomer-like state in the SURMOF and solution, respectively. Using the values for the lifetimes presented above and the reasonable assumption that $\eta_{\text {solution }}^{\text {int }} \approx 100 \%$, we can calculate estimates for the internal PLQY based on the kinetics of roughly $14 \pm 0.5$, $12 \pm 0.3$, and $8 \pm 0.2 \%$ for the 10,50 , and $100 \%$ ADB samples, respectively. These PLQYs calculated from the kinetics agree reasonably well with the observed values, and allow us to conclude that a higher yield of aggregate formation at higher concentrations of $\mathrm{ADB}$ is the physical process that mainly limits the PLQY in these systems.

Examining Figure 2 further, we will briefly discuss the effect of the changing fraction of ADB on the external PLQY, which is defined as the number of emitted photons divided by the number of photons incident on the sample. It is a figure-ofmerit of interest for many practical applications where the total brightness of the sample under a given illumination is important. The external PLQY is equal to the internal PLQY multiplied by the fraction of photons absorbed by the sample. Therefore, realizing a high external PLQY required the sample to exhibit not only a high internal PLQY but also strong absorption. Figure $2 \mathrm{~b}$ illustrates that the absorption strongly decreases as the fraction of ADB within the SURMOF is reduced. This indicates that the external PLQYs are similar and below $1 \%$ for all three ratios, namely $0.66 \pm 0.1,0.88 \pm 0.44$, and $0.68 \pm 0.51 \%$ for the 10,50 , and $100 \%$ ADB samples, respectively.

To summarize the results of the first strategy to enhance the SURMOF PLQY, the dilution of the ADB linker with an inert linker was found to increase the internal PLQY by a factor of two at a fraction of $10 \%$. However, when the absorp- tion is concurrently reduced, there is little gain in the external PLQY. In addition, further reduction in the ADB fraction are not desirable as the very small fraction of active linker in the SURMOF would compromise both absorption and excitedstate transport properties (these negative effects outweighing the likely better PLQYs in terms of practical applications). In the next section, we tackle the problem of increasing the PLQY while maintaining absorption and excited-state transport properties by using a second strategy: the introduction of acceptor chromophores in an ADB matrix.

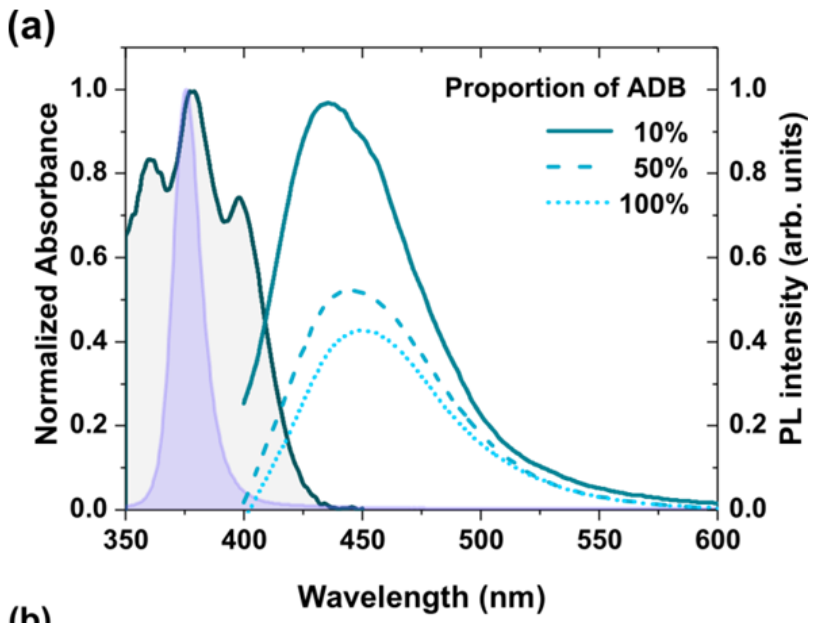

(b)

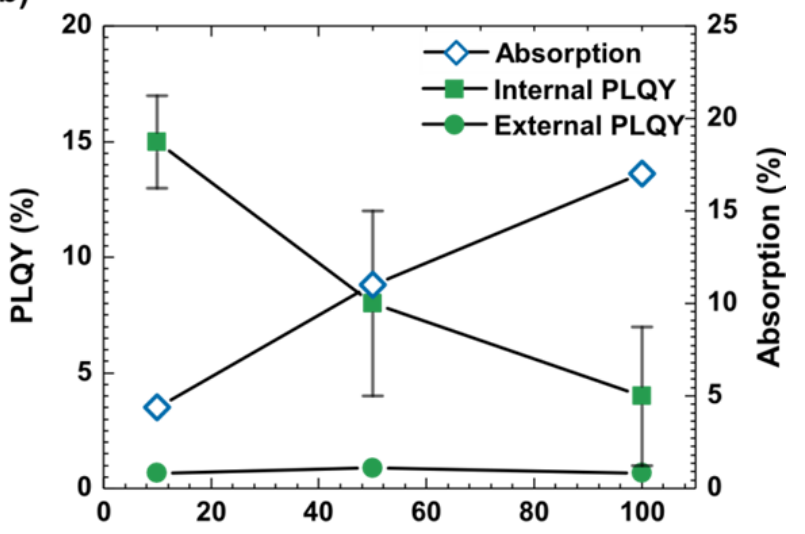

Proportion of ADB in linker solution (\%)

Figure 2 a) Steady-state PL spectra of the SURMOFs with varying $\mathrm{ADB}$ fractions together with the absorption spectrum of the ADB linker and the spectrum of the LED excitation source (375 $\mathrm{nm})$ that was used for the steady-state PL and PLQY measurements. The PL spectra are normalized to the maximum internal PLQY, to demonstrate the difference efficiencies of emission between the samples. b) The internal PLQY, sample absorption, and external PLQY as a function of ADB linker proportion in the linker solution.

\section{Coumarin dyes infiltrated into ADB SURMOF}

In this second strategy, we constructed SURMOFs based on only ADB linkers and added a varying amount of one of two coumarin derivatives into the linker solution: a given concentration of either coumarin 343 (C343) or coumarin 334 (C334) was added to the ADB linker solution. The overlap between the emission spectrum of the ADB SURMOF and absorption spectrum of $\mathrm{C} 343$ and C334 suggest that energy transfer should be efficient (see Figure S3). The main difference be- 
tween C343 and C334 is that C343 has a carboxylic group whereas the C334 has a methyl ketone group in position 10 . We hypothesized that the carboxylic group of C343 will lead to strong interaction within the SURMOF by the formation of hydrogen bonds. This was confirmed by infrared absorption spectroscopy (see Figure S4). Further evidence for a noncovalent bonding of the C343 molecule within the SURMOF was realized by washing a C343-containing SURMOF via immersion in a solvent (such as toluene and ethanol) for three days. Although some of the $\mathrm{C} 343$ was washed out (leading to a slight blue-shift of the PL emission spectrum) most stays within the SURMOF supporting the conclusion that hydrogenbonding secures the guest $\mathrm{C} 343$ molecules within the SURMOF (see Figure S5). Figure 3a schematically illustrates these two cases. C343 is sketched as a green molecule that interacts with the SURMOF via the carboxylic group. C334 is shown in Figure $3 \mathrm{a}$ as a red molecule that does not form any hydrogen-bonds with the SURMOF. The sample is rinsed with ethanol in each synthesis cycle in the layer-by-layer deposition approach, which means that it is possible that a significant fraction of the C334 is washed out of the SURMOF. Using these two dyes allows us to clearly demonstrate that C343 is indeed interacting strongly with the coordination network, and show that the concentration of C343 inside the SURMOF can be controlled reliably. We note that a completely fabricated SURMOF can be loaded with C343 molecules by dropcasting a $20 \mu \mathrm{M}$ solution ethanolic solution of $\mathrm{C} 343$ onto a pure ADB film (see Figure S6). However, the post-fabrication loading method lead to less control of the concentration of the C343 molecules in the ADB SURMOF so we utilize the loading during SURMOF construction technique in the rest of this work.

We fabricated samples using nine different molar ratios between the $\mathrm{C} 343$ or $\mathrm{C} 334$ guests and the ADB linker (30, 20, $10,5,2,1,0.1,0.02,0.005 \%$ concentrations of the guest relative to the linker). Figure $3 \mathrm{~b}$ illustrates the absorption and emission spectra of each of these SURMOFs. For comparison, we plot the absorption and emission spectra of C343 and C334 solutions as filled curves in Figure 3b. Examining the C343 data in Figure $3 \mathrm{~b}$, we see that the SURMOF emission peak correlates with the proportion of the dye in the linker solution. The emission of the $0.005 \%$ sample is still influenced by emission from the ADB, leading to an emission-maximum that is blue-shifted with respect to that of the C343 in solution. However, as the C343 concentration is increased the absorption peak at $440 \mathrm{~nm}$ steadily grows and the emission spectrum steadily shifts towards the red. The red-shifting of the PL spectrum with increasing concentration could be due to increasing reabsorption of the blue $\mathrm{C} 343$ emission due to the growth of the absorption band centered at $440 \mathrm{~nm}$ plus some solvatochromic effect due to the hydrogen-bonding of the C343 chromophore red-shifting its emission energy (as has been observed for solvent environments). ${ }^{35}$

In contrast, the ADB SURMOFs containing C334 (shown in red in Figure $3 b$ ) behave significantly differently. Firstly, irrespective of the $\mathrm{C} 334$ concentration in solution, there is no absorption peak at $440 \mathrm{~nm}$ in the SURMOF; the absorption spectrum of the SURMOFs remain unchanged over all the C334 concentrations. This indicates that significantly less C334 is taken up by the SURMOF. Although the emission spectrum is slightly altered by the small concentration of C334 guest molecules in the SURMOF, there is no direct correlation between the maximum of the PL emission spectrum and the concentration of C334 in solution. The amount of C334 retained as guest molecules in the SURMOF does not correlate with the concentration in solution, and therefore likely is influenced by the washing procedure. Figure $3 \mathrm{c}$ shows the wavelength position of the PL maximum for SURMOFs with C343 and $\mathrm{C} 334$ as a function of dye concentration in the initial solution. In the case of $\mathrm{C} 343$, clear evidence of a controlled introduction into the SURMOF is obtained as the peak steadily shifts. However, in the case of $\mathrm{C} 334$, the amount of dye uptake fluctuates, most likely due to an uncontrolled dependence on a varying amount of dye washed out in the rinsing cycle more than on the concentration of the dye in the sprayed solution. Comparing these data sets, we conclude that we are able to introduce defined amounts of $\mathrm{C} 343$ into the ADB SURMOF by controlling the amount of $\mathrm{C} 343$ in the linker solution.

We next studied the PLQY in the donor-acceptor mixed SURMOFs with varying concentrations of C343 (see C334 in Figure S7). The PLQY of C343 in solution is $63 \% .{ }^{36}$ Figure $4 \mathrm{a}$ shows the dependence of the PLQY as a function of the concentration of $\mathrm{C} 343$ in the linker solution. ADB SURMOFs containing C343 (C343@ADB) demonstrate a very interesting behavior. When the amount of dye is reduced from $30 \%$ to $1 \%$ the PLQY increases, reaching a maximum of $50 \pm 2 \%$ (average of three independently-prepared samples). We attribute the increase in PLQY with reducing C343 concentration in this regime to the reduction in reabsorption and interactions between C343 molecules as their fraction in the matrix decreases. 
(a)
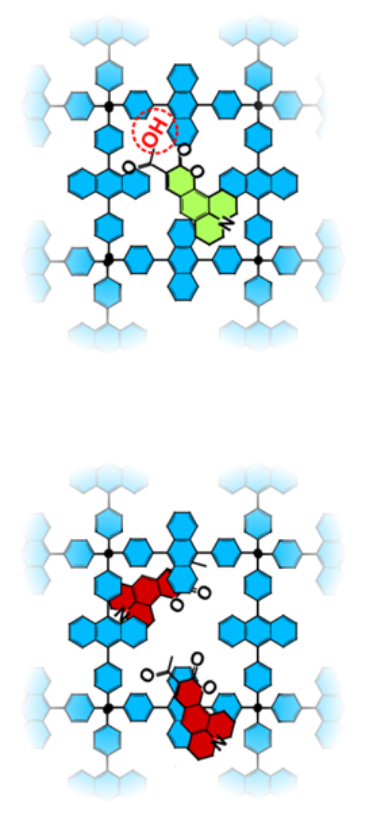

(b)

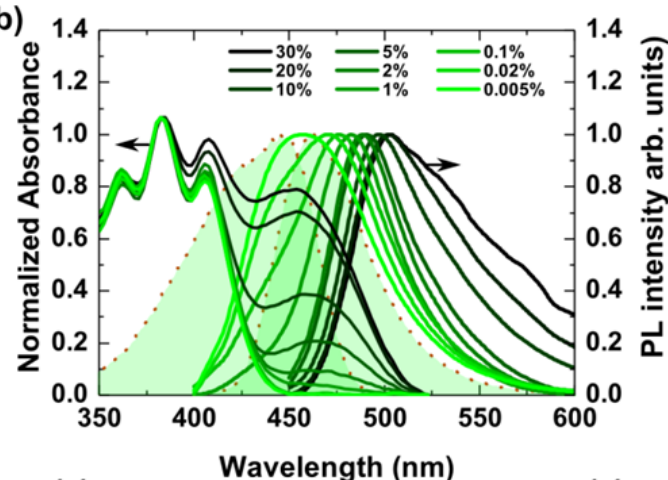

(c)

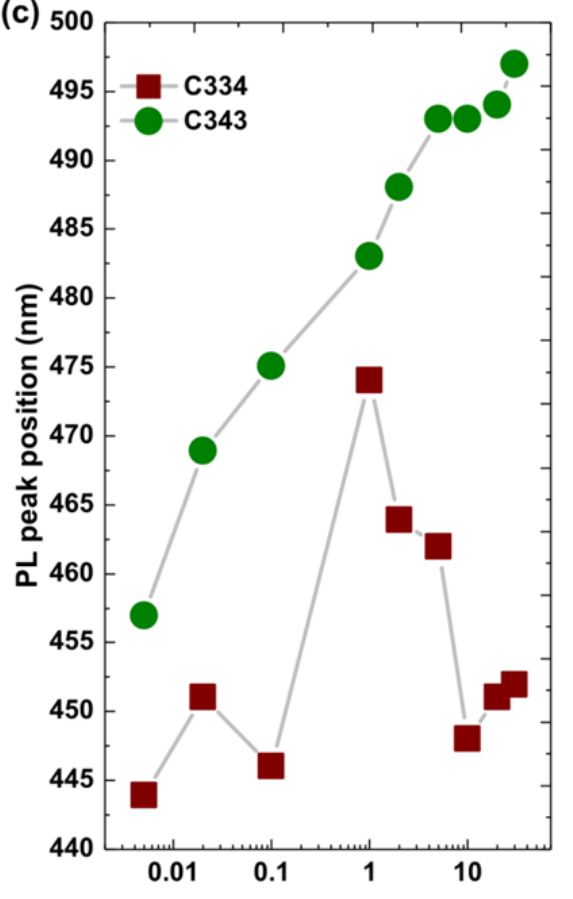

Proportion of dye in linker solution (\%)

Figure 3. (a) Schematic drawings of ADB SURMOFs that are infiltrated with coumarin 343 (C343, green colored molecule) (upper drawing) and with coumarin 334 (bottom drawing, red colored molecule). The dashed red line illustrates the interaction of C343 with the ADB SURMOF. Panel (b) shows the absorption and emission spectra of C343 and C334 solutions, both as filled curves. The solid curves show the absorption and emission spectrum of ADB SURMOFs infiltrated with different concentration of C343 (top) or C334 (bottom). (c) The peak position of the PL emissions is plotted in dependence of the fraction of C343 and C334 in the linker solution.

We note that at $1 \%$ loading the XRD spectrum is unchanged from the pure ADB sample. As the amount of C343 is reduced below $1 \%$, the PLQY starts to decrease with decreasing concentration, reaching $28 \%$ for a concentration of $0.005 \%$. The reduced PLQY at very low concentrations can be understood in terms of a reduced efficiency of energy transfer when the acceptor dyes are so sparse. At low concentrations of acceptor, there are volumes in the sample from which the emission must come from the ADB donor, as the excited state on the donor cannot reach an acceptor within its lifetime. The combination of both effects, high reabsorption for high concentrations and low energy transfer efficiency for low concentrations lead to an optimum value, which is in this case at $\sim 1 \%$ C343 dye in the ADB linker solution. To verify the reproducibility of this approach we prepared three samples at each concentration of $2 \%, 1 \%$ and $0.1 \%$ and found that the maximum was always at $1 \%$ of C343 (see Figure S8).

\section{Singlet exciton dynamics within ADB SURMOF}

Even when the concentration of C343 is low, its effect on the PL emission spectrum and PLQY is still observable and systematic. This suggests that energy can move rapidly within the ADB SURMOF. A first rough approximation of the diffusion length can be made by assuming diffusion occurs primarily in the direction of the $0.6 \mathrm{~nm}$ spacing between linkers on adjacent sheets. Given the PLQY of the SURMOF constructed from the solution with $1 \%$ coumarin is roughly equivalent to the PLQY of coumarin in solution, we can approximate that all excitations reach an acceptor in this SURMOF. We note that this is consistent with the recent results using coumarin acceptors in truxene MOFs wherein over $90 \%$ energy transfer efficiency was also observed for a $1 \%$ coumarin doping level. ${ }^{11}$ We can use the high level of quenching in this SURMOF fabricated with the $1 \%$ coumarin solution to estimate the diffusion lengths of excited-states on the ADB linkers in these SURMOFS. Based on the nearest neigbour separation of $0.6 \mathrm{~nm}$ and assuming that the $\mathrm{C} 343$ remains at a $1 \%$ dilution as it was in the solution, we estimate a separation of acceptors on the linear row of nearest neighbor chromophores of about $60 \mathrm{~nm}$. Therefore, we approximate a diffusion length of roughly half this distance, namely $30 \mathrm{~nm}$. We note that the ratio of linkers in the SURMOF could well be different from the known ratio in solution, and is not easy to quantify. We can estimate the ratio of the donor and acceptor molecules in the SURMOF by evaluating the ratio of the ADB absorption peak at $360 \mathrm{~nm}$ and the $\mathrm{C} 343$ absorption peak at $450 \mathrm{~nm}$ in the SURMOF and referencing this absorption ratio to a solution of known concentration (see Figure S9). From this analysis, we obtain that the sample containing $1 \%$ of $\mathrm{C} 343$ in the linker solution results in SURMOF with $0.6 \%$ of $\mathrm{C} 343$ in the SURMOF, which would increase the average separation to $100 \mathrm{~nm}$. Therefore, our rough estimate of the diffusion length in our system give values in the range of 30 to $50 \mathrm{~nm}$. Such an exciton diffusion length is long compared to many organic semiconductors, ${ }^{37-39}$ but comparable to singlet diffusion lengths reported chromophores ordered in nanochannels, ${ }^{40}$ and for anthracene crystals. ${ }^{41}$ Furthermore, it is in good agreement with the more rigorously determined singlet exciton diffusion lengths of $48 \mathrm{~nm}$ for the truxene MOF system, ${ }^{11}$ and also the net motion of excitons $52 \mathrm{~nm}$ in a porphyrin-based MOF (where we calculate this length from the reported average absolute displacement of 45 linkers and the nearest-neighbor separation of $1.15 \mathrm{~nm}) .^{13}$ 
(a)

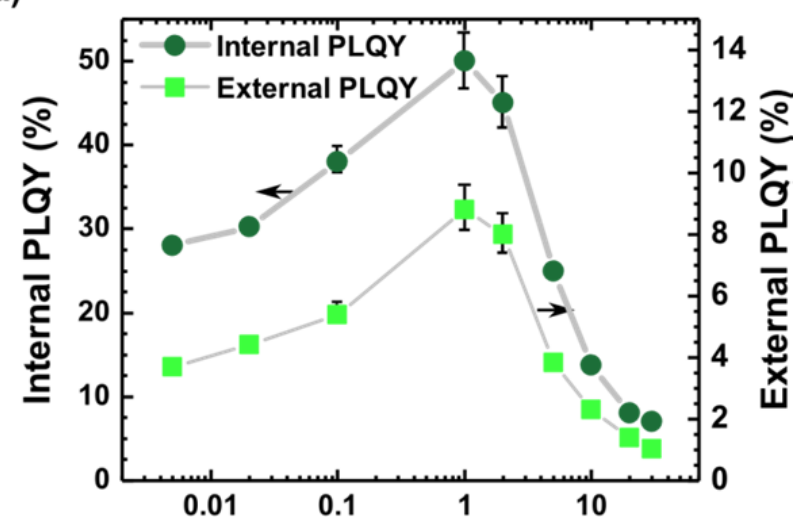

Proportion of C343 in linker solution (\%)

(b)

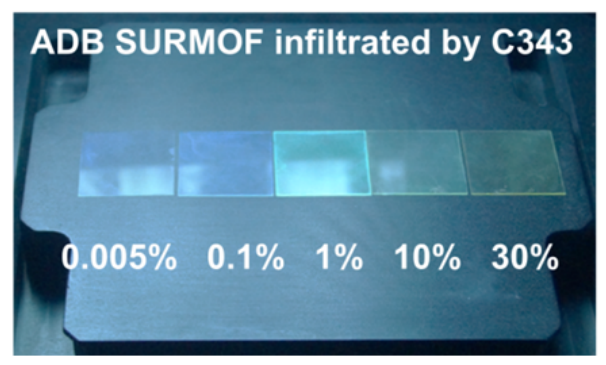

Figure 4. (a) The internal and external PLQY of ADB SURMOFs infiltrated by $\mathrm{C} 343$ as a function of the concentration of the dye in the ADB linker solution. Samples with $2 \%, 1 \%$ and $0.1 \%$ of C343 were prepared and investigated three times to estimate the error, which is indicated by the error bar. (b) Photo of five ADB SURMOFs samples with C343 upon illumination by a UV lamp $\left(\lambda_{\mathrm{ex}}=375 \mathrm{~nm}\right)$. The concentration of $\mathrm{C} 343$ in solution relative to the ADB linker from left to right is $0.005,0.1,1,10$, and $30 \%$.

To look at the motion of excited-state species in the ADB SURMOF in more detail, we examined the excited-state annihilation in the pure ADB SURMOF. We recorded the timeintegrated emission spectra as a function of excitation fluence using $400 \mathrm{~nm}$ femtosecond pulses. This allows us to analyze the mobility of the excited-state species through the reduction of the emission efficiency due to singlet-singlet annihilation (SSA) at higher intensities. Figure 5a demonstrates that the emission spectrum blue-shifts as the excitation fluence increases. This is explained by the redder aggregate emission being quenched relative to the short-lived blue monomer-like emission at higher fluences. This suggests that there is more annihilation between the longer-lived aggregate species than the monomer-like species and that they therefore likely contribute to the transport of energy to the C343. The overlap between the emission of the ADB aggregate state and the absorption of the C343 is shown in Figure S3.

To analyze the motion of excited states in the ADB in more detail, the time-integrated PL intensity as a function of excitation intensity is plotted for both the aggregate and monomer-like species in Figure 5b. Both emissions of monomerlike species (emission integrated from $440-460 \mathrm{~nm}$ ) and the aggregate state (emission integrated from 550-750 nm) shows a sublinear dependence on the excitation fluence. This indi- cates that both species move and annihilate during their lifetimes.

(a)

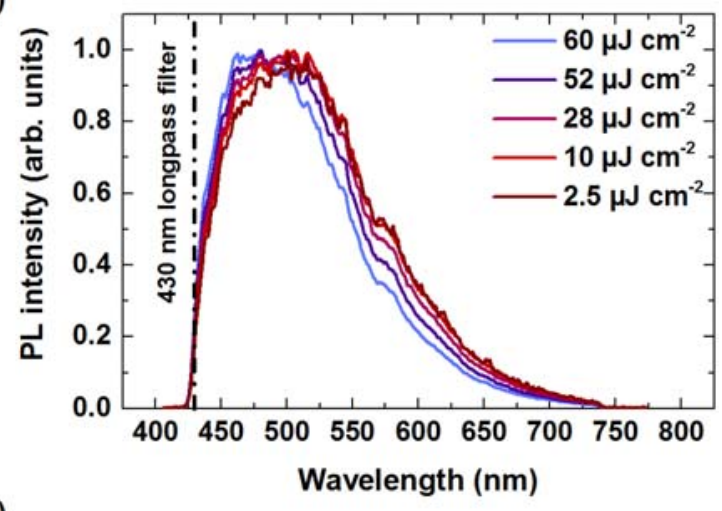

(b)

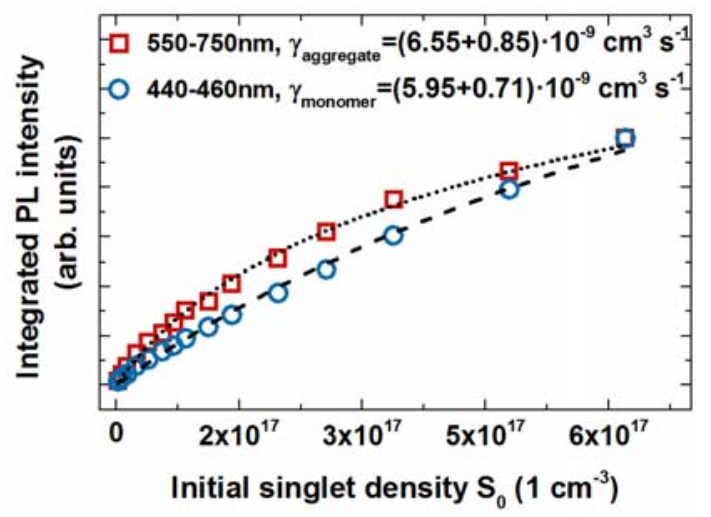

Figure 5. (a) Normalized time-integrated emission spectra of a pure ADB SURMOF excited with different pulse energy densities. (b) Spectrally integrated PL intensities (normalized to maximum) as functions of the initial singlet density $S_{0}$, which is further explained in the text. The data were fit to determine ratio of the bimolecular annihilation coefficient to the monomolecular decay rate, with the estimates of the monomolecular decay rates from above, the bimolecular annihilation coefficient can be determined for the aggregate and monomer state (see text).

We note that absorption saturation does not contribute to the nonlinearity; the absorption as a function of fluence remains linear as shown in the SOI (see Figure S10). In order to extract information about the excited-state diffusion from these data, one can express the decay of the monomer and aggregate state by a mono and bimolecular term:

$$
\frac{\partial A}{\partial t}=-k A-\gamma A^{2}
$$

where $A$ is the excited-state density, $k$ the monomolecular recombination constant and $\gamma$ the bimolecular recombination constant. In the case of three-dimensional diffusion, $\gamma$ can be accurately approximated as being time-independent. With $A(t=0)=A_{0}$ as the initial aggregate state population the solution for the above rate equation is (see section S VIII for calculation details):

$$
A_{3 D}(t)=\frac{k A_{0}}{\exp (k t)\left(k+\gamma A_{0}\right)-\gamma A_{0}} .
$$

The recorded emission intensity for a given excitation intensity corresponds to the integral of this solution over time and the following analytical expression for this can be derived: 


$$
\int_{0}^{\infty} k A_{3 D}(t) d t=\frac{k}{\gamma} \ln \left(1+\frac{\gamma}{k} A_{0}\right),
$$

where $A_{0}$ is the intitial density of aggregate states and can be estimated as the total number of photons absorbed. This slight overestimate neglects the minority of excited-states that decay to the ground state via monomer-like species, which will lead to a slight underestimate of the diffusion length of the aggregate. The initial excitation density can be calculated from the pump fluence, the fraction of excitation photons absorbed (7\%), and the film thickness $(140 \mathrm{~nm})$.

The data can be fitted with the expression derived, which has the ratio $\gamma / k$ as the only fitting parameter. We fit equation (4) to the data shown in Figure $5 \mathrm{~b}$ and extracted for the aggregate state $\gamma / k=(2.16 \pm 0.14) \cdot 10^{-17} \mathrm{~cm}^{3}$ and for the monomer state $\gamma / k=(0.22 \pm 0.05) \cdot 10^{-17} \mathrm{~cm}^{3}$. Knowing that $k$ is the inverse of the lifetime from Figure $1 \mathrm{~b}$ and $1 \mathrm{c}$ for the monomer $(0.37 \mathrm{~ns})$ and for the aggregate emission (3.3 ns), we calculate the bimolecular recombination rates to be $\gamma_{\text {aggregate }}=(6.55 \pm 0.85) \cdot 10^{-9} \mathrm{~cm}^{3} / \mathrm{s}$ and $\gamma_{\text {monomer }}=$ $(5.95 \pm 0.71) \cdot 10^{-9} \mathrm{~cm}^{3} / \mathrm{s}$ The bimolecular recombination rate is related to the diffusion length $L_{3 D}=\sqrt{6 D / k}$ (assuming three-dimensional diffusion) ${ }^{39}$ by:

$$
\gamma=\frac{4}{3} \pi k R_{0} L_{3 D}^{2}
$$

where $R_{0}$ is the effective interaction radius of the annihilation. This gives us an estimate for the the aggregate state $R_{0} L_{3 D}^{2}=$ $5200 \pm 300 \mathrm{~nm}^{3}$ and for the monomer state $R_{0} L_{3 D}^{2}=525 \pm$ $120 \mathrm{~nm}^{3}$

As the time-resolved emission strongly suggests that the aggregate states spread at least over two ADB molecules, we estimated $R_{0}$ to be at least $2 \mathrm{~nm}$, giving us a diffusion length of at most $L_{3 D}^{\text {aggregate }}=51 \pm 2 \mathrm{~nm}$ For the monomer state we assumed that the interaction radius should be restricted to single ADB molecules, $R_{0} \sim 1 \mathrm{~nm}$. This results in a diffusion length of $L_{3 D}^{\text {monomer }}=23 \pm 3 \mathrm{~nm}$ for the monomer state. These diffusion lengths agree well with the earlier diffusion length rough estimation from the PLQY measurements, suggesting that both the monomer-like and aggregate state can transport energy to the $\mathrm{C} 343$ acceptors.

However, the assumption of three-dimensional diffusion may well not be justified because the non-isotropic arrangement of dyes within the SURMOF could well lead to preferential energy transport into a certain direction. For the ADB SURMOF the lattice constant into (100) and (101) direction is $2.2 \mathrm{~nm}$ but is $0.6 \mathrm{~nm}$ into (010) direction. ${ }^{25}$ This consideration makes a preferable one-dimensional transport in the (010) direction likely. In the case of one-dimensional transport $\gamma$ is time dependent: ${ }^{42}$

$$
\gamma=4 \pi D R_{0} \frac{R_{0}}{\sqrt{2 \pi D t}}
$$

Solving the rate equation with this time dependent $\gamma$ leads to following solution:

$$
A_{1 D}(t)=\frac{A_{0} \exp (-k t)}{2 \pi L_{1 D} R_{0}^{2} A_{0} \operatorname{erf}(\sqrt{k t})+1},
$$

with $\operatorname{erf}(x)$ being the error function and $L_{1 D}=\sqrt{2 D / k}$ the one-dimensional diffusion length. There is not an analytic for the integral $\int_{0}^{\infty} k A_{1 D}(t) d t$ expression, but the integral can be taken numerically and input into a non-linear regression meth- od to fit the parameter $L_{1 D} R_{0}^{2}$ ( $k$ is kept constant at the known value determined by the inverse lifetime). Performing the nonlinear regression leads to $L_{1 D} R_{0}^{2}=655 \pm 20 \mathrm{~nm}^{3}$ for the aggregate state and $L_{1 D} R_{0}^{2}=$ $145 \pm 23 \mathrm{~nm}^{3}$ for the monomer state.

Here we see that the estimate of dimension length for onedimensional diffusion depends more strongly on the interaction radius. ${ }^{38,42}$ Assuming interaction radii $\left(R_{0}\right)$ of $1,2,3$ and 4 $\mathrm{nm}$ would result in diffusion lengths $L_{1 D}^{\text {aggregate }}$ of 655,163 , 72 , and $41 \mathrm{~nm}$ respectively for the aggregate state. For the monomer state the corresponding diffusion lengths $L_{1 D}^{\text {monomer }}$ are 145, 36, 16 and $9 \mathrm{~nm}$. The latter increased interaction radius results in a diffusion length that seems the most physically reasonable.

At this stage, we conclude that the significant transport lengths that underlie the improved PLQY in the C343 guest samples are achieved due to good exciton diffusion, both in the monomer and in the aggregate state. Additionally, a large interaction radius may play a role, which is due to either delocalization of the excited states or the possibility of longerrange interaction of the exctited-states through a Förster mechanism (FRET) as found for the truxene MOFs. ${ }^{11}$

The precise details explaining the physical basis for energy transfer in MOFs are an interesting area for further exploration. For exciton transport through aggregates, a controlled position of the molecules in space is critical. ${ }^{43}$ Such control has been previously been shown to enhance the efficiency of organic photovoltaic devices. ${ }^{44}$ Similarly, crystalline order is known to be critical for achieving long diffusion lengths. ${ }^{45}$ SURMOFs therefore should provide a platform wherein excited-state diffusion can be accurately adjusted, and long diffusion lengths engineered. Additionally, investigation of the role of reduced dimensionality in the energy transfer processes due to the asymmetry in the lattice directions is of further interest.

\section{Utilization of C343@ADB in an upconversion SURMOF heterostructure}

In order to test the efficiency of the newly developed C343@ADB SURMOF, we used it as an emitter layer in a photon UC SURMOF heterostructure wherein the sensitizer layer was built with a Pd-porphyrin based linker [(5,10-bis(4carboxyphenyl)-10,20-diphenylporphyrinato)palladium(II)] (Pd-DCP). We have previously shown triplet states can be transferred from the sensitizer to emitter SURMOF layers in order to allow triplet-triplet annihilation-based photon upconversion (TTA-UC). ${ }^{25}$ Hitherto the emitter layer was a pure ADB SURMOF, here we use instead the C343@ADB in the hope of significantly increasing the UC PLQY.

The SURMOFs were deposited via a spin-coating technique, wherein one deposition cycle consists of spin-coating a metal center solution $(0.2 \mathrm{mM})$ followed by the linker solution $(20 \mu \mathrm{M}){ }^{46}$ To prepare the bilayers, after a given number of cycles of the emitter layer, the linker solution was changed to the sensitizer and a further number of cycles of the sensitizer SURMOF were grown onto the emitter SURMOF. This spincoating method leads to SURMOFs with lower surface roughness as compared to spray-coated SURMOFs previously used, ${ }^{25}$ as observed by scanning electron microscopy (Figure S11).

We created four samples, one C343@ADB SURMOF (10 cycles) with $1 \%$ C343 in the linker solution, one pure Pd-DCP SURMOF (10 cycles) and two bilayers in which the first layer 
is the 1\% C343@ADB SURMOF (10 cycles) and the second layer is a Pd-DCP SURMOF made from either 4 or 8 cycles. Figure 6a shows the coplanar XRD of the Pd-DCP SURMOF, the C343@ADB SURMOF and the Bilayer (8 cycles PdDCP). The position of the XRD scattering peaks in the bilayer agrees well with a sum of the scattering peaks coming from its individual layers. ${ }^{27}$ This indicates the structural quality of the individual layers are maintained in the bilayer samples. The Pd-DCP shows the first order of the (001) orientation at $3.7^{\circ}$. Using the Bragg condition, this translates to a lattice constant of $2.4 \mathrm{~nm}$. The C343@ADB (1\%) SURMOF shows the first order of the (001) at around $4.1^{\circ}$ corresponding to a lattice

(a)

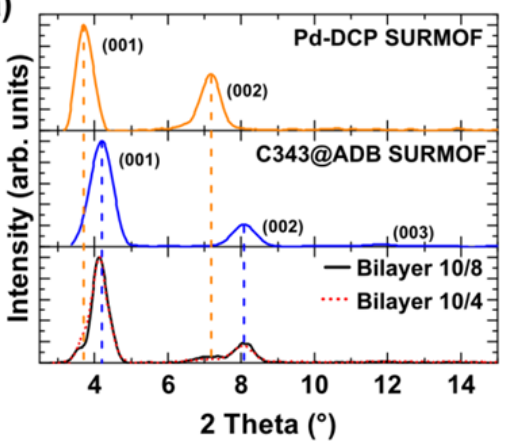

(c)

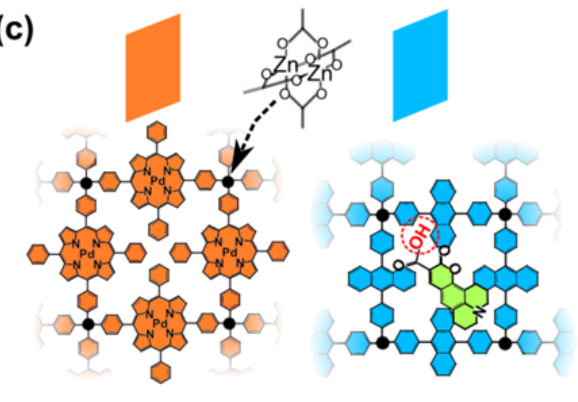

(b)
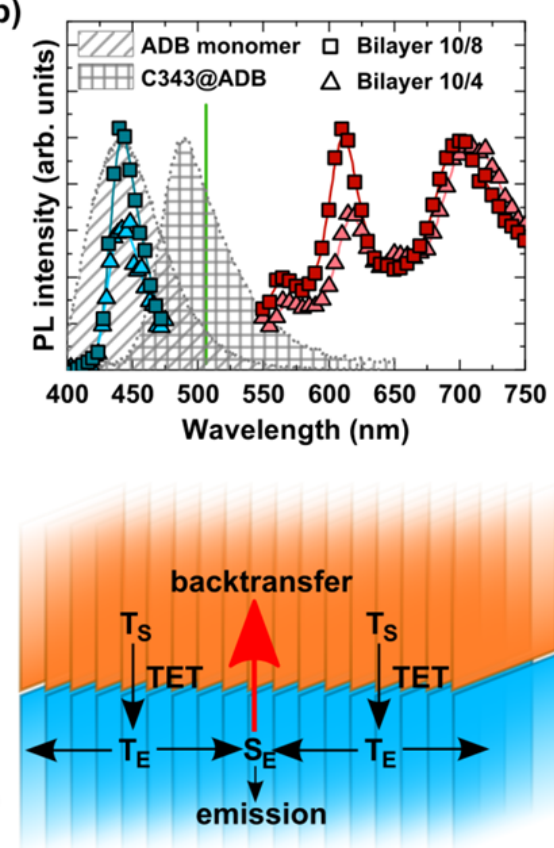

constant of $2.2 \mathrm{~nm}$. Using the Scherrer equation and the width of the first order XRD peaks, it is possible to estimate the growth rate of each SURMOF. For the C343@ADB, a growth rate of $\sim 2 \mathrm{~nm}$ per cycle was inferred and for the Pd-DCP a growth rate of $\sim 2.4 \mathrm{~nm}$ per cycle. It is worth mentioning, that the spin-coating gives more precise control over the SURMOF thickness than the spraying technique, where a faster growth rate of 10-15 $\mathrm{nm}$ per cycle was inferred. The C343 is also incorporated into the SURMOF in the spin-coating fabrication technique, as confirmed by optical absorption measurements ofC343@ADB SURMOF samples (Figure S12).

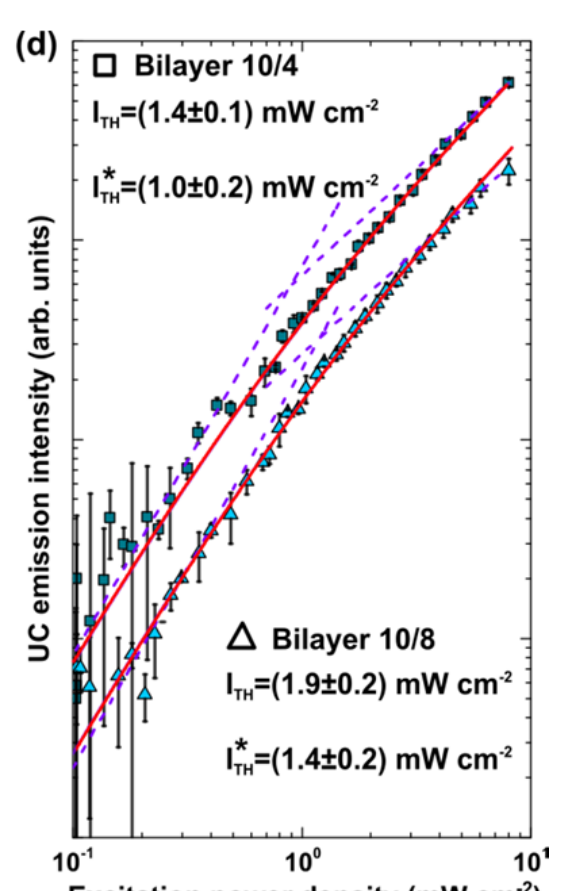

Excitation power density $\left(\mathrm{mW} \mathrm{cm}^{-2}\right)$

Figure 6. (a) Coplanar XRD showing the crystallinity and orientation of the Pd-DCP SURMOF, the C343@ADB SURMOF and the bilayer made from C343@ADB SURMOF and 8 cycles of Pd-DCP SURMOF. The peaks are labeled with the orientation. Additionally, the dashed lines indicate the composition of the bilayer from the monolayers. (b) PL emission spectrum of the two bilayers together with the emission spectra of ADB monomer emission and C343@ADB emission as filled curves. (c) Schematic of the SURMOF heterostructure bilayers together with the flow of triplet excitons across the interface. The sensitizer triplets, TS, generated in the Pd-DCP SURMOF (orange sheets) can reach the interface and transfer across it to create emitter triplet states, TE, on the C343@ADB SURMOF (blue sheets). Assuming the predominance of one-dimensional diffusion the TE states will stay near the interface, and upon their annihilation the singlet emitter, SE, can have a high probability to transfer energy back to the Pd-DCP SURMOF. (d) Intensity dependence measurements for the UC emission of both bilayers together with the analytic fit (solid lines) and the approximated asymptotes (dashed lines) with a slope of two and one respectively. Additionally, thresholds from the analytic fit and the approximated asymptotes (indicated by an asterisk) are noted.

Figure $6 \mathrm{~b}$ shows the emission spectra of the two bilayers using a $532 \mathrm{~nm}$ continuous-wave laser excitation source. The emission above $550 \mathrm{~nm}$ comes from the sensitizer Pd-DCP SURMOF. However, The UC emission below $500 \mathrm{~nm}$ is due to triplet-triplet annihilation in the emitter C343@ADB SURMOF. Surprisingly, the peak of the emission does not coincide with the peak position of the directly-excited C343@ADB (1\%) SURMOF, shown as a cross-hatched curve in Figure 6b. Instead, the peak position agrees well with the emission peak of a pure ADB monomer emission, which is shown as a single-hatched curve. However, if the C343@ADB (1\%) emitter layer that is part of a bilayer is directly excited (at $370 \mathrm{~nm}$ ) the maximum of the PL spectrum is only slightly shifted to the blue as compared to that of a normal
C343@ADB (1\%) layer (see Figure S13). Therefore, there is a clear difference between the emission spectrum of the C343@ADB (1\%) bilayer when it is excited via triplet-triplet annihilation versus when it is excited by normal photon absorption.

To explain this difference in emission spectrum between direct excitation and excitation via triplet-triplet annihilation we hypothesize that the distance of the excited-states from the sensitizer interface and FRET transfer of the singlet state to the sensitizer layer play key roles. We hypothesize that, due to the exponential falloff of the Dexter rate with separation, the triplet transport in the emitter layer is significantly easier between sheets $(0.6 \mathrm{~nm}$ separation between chromophores) than within a single sheet $(2.2 \mathrm{~nm}$ separation between chro- 
mophores). As schematically shown in Figure 6c, this means that triplets will not move away from the interface but rather stay in the portion of the emitter layer close to the sensitizer layer. Thus, the singlets created by triplet-triplet annihilation will also be close to the interface, and easily able to transfer back to the sensitizer through FRET if there emission overlaps with the sensitizer absorption. As shown in Figure S14, the absorption of the Pd-DCP SURMOF starts to increase at 510 $\mathrm{nm}$ overlapping well with the aggregate and C343 emission. This would allow excited-states that move to the C343 acceptor to subsequently move back to the sensitizer; the efficient transfer back to the sensitizer from the C343 effectively quenching its emission. The few monomer-like states on the $\mathrm{ADB}$ that emit before energy is transferred to a redder state would therefore be responsible for the weak upconversion that is observed.

When excitons are created through the entire thickness of the emitter layer by direct optical excitation of the emitter, the spectrum of the emitter layer of the bilayers is slightly shifted to the blue as compared to the lone 1\% C343@ADB SURMOFs. This also suggests that although there is a large contribution to the emission in the bilayer's emitter from C343 that are far enough away from the interface that they are not efficiently quenched by back-transfer, C343 excited-states are more effectively quenched by back-transfer than monomerlike states (thus explaining shift between the PL spectra). To further examine this hypothesis, we collected the timeresolved emission of the bilayers using the Streak camera setup (see Figure S15). Using a $380 \mathrm{~nm}$ excitation wavelength, we directly excited the ADB of the bilayers and the pure C343@ADB emitter layer. At emission wavelengths from 470-510 nm (corresponding to PL from C343), the PL lifetime of the bilayer is significantly shorter than that of the C343@ABD. This indicates that the C343 excited-states in the bilayer can indeed be quenched by transferring back onto the sensitizer layer. The PL from 410-450 nm, stemming from the monomer-like ADB states, is not as strongly quenched; the lifetime in the bilayer is only slightly shorter than in the C343@ABD alone. These observations are consistent with the hypothesis that the upconverted emission comes from a small amount of emission from the monomer-like state before it can transfer to C343. Unfortunately, the C343 emission - that would be much stronger - is not observed due to the fast return of the excited-state on the C343 back to the sensitizer layer.

Although this back transfer of singlet excitons drastically reduces the quantum efficiency of the upconversion emission, it has little effect on the upconversion threshold. ${ }^{47}$ The data measured for the UC intensity as a function of excitation intensity are shown in Figure 6d. These data were fitted with a theoretical curve for the intensity dependence derived from the rate equations for TTA-UC (albeit in a homogeneous solution). ${ }^{47}$ Additionally, this approach is compared with estimation of the threshold by overlaying the low-intensity data points with a line whose slope is 2 and the high intensity data points with a line whose slope is 1 . The threshold can then be approximated as the intensity at which these two lines cross. The bilayer with the thinner Pd-DCP layer has a threshold of $1.9 \pm 0.2 \mathrm{~mW} \mathrm{~cm}^{-2}$ extracted by the fit to the analytic curve and a threshold of $1.4 \pm 0.2 \mathrm{~mW} \mathrm{~cm}{ }^{-2}$ extracted by the intersection of the two asymptotes. In comparison, the bilayer with the thicker Pd-DCP SURMOF shows a threshold of $1.4 \pm 0.1 \mathrm{~mW}$ $\mathrm{cm}^{-2}$ extracted by the fit and a threshold of $1.0 \pm 0.2 \mathrm{~mW} \mathrm{~cm}$ extracted by the two asymptotes. These low thresholds are consistent with our previous observations, ${ }^{25}$ and the low thresholds measured in layered structures by Hanson et al. ${ }^{48}$

In order to achieve high quantum efficiency with the low threshold, further work is necessary to better engineer the excited-state energy flow and create a greater distance between the singlet states created by triplet-triplet annihilation in the emitter and the sensitizer-emitter interface. For example, a first promising strategy would be to rotate the SURMOF sheets with respect to the interface, so that the triplet transport towards and away from the interface is faster. In addition, using a bridging layer between the sensitizer and emitter layers that would allow triplet transport but suppress singlet exciton back-transfer could increase the quantum efficiency.

\section{Conclusions}

We studied two approaches for enhancing the luminescent properties of thin film ADB SURMOFs. Though the PLQY of ADB SURMOFs is low due to the formation of aggregate-like states, we found that the PLQY can be enhanced by fabricating a SURMOF in which the ADB linker is diluted with an optically inert linker, or by introducing C343 as an acceptor molecule in a pure ADB SURMOF. The first strategy was effective in raising the internal PLQY, but at the cost of lowering the absorption and the excited-state transport properties. The second strategy led to enhancement of the internal PLQY and external PLQY without sacrificing excited-state transport properties.

By varying the concentration of $\mathrm{C} 343$ we obtained an optimized internal PLQY of $52 \%$, approaching the $63 \%$ of the C343 linker in solution. This result implies good excited-state energy diffusion in the donor (ADB) molecules in order that excited states can reach the acceptors (C343). Investigation of the intensity dependence of the PL of a pure ADB SURMOF revealed that the aggregate-like states have a longer diffusion length than the states responsible for the monomer-like emission. This suggests that the aggregate states may be the main long-distance energy carrier in the SURMOF, and play a role in bringing energy to the $\mathrm{C} 343$ acceptors.

Energy transport in SURMOF structures is of on-going interest. The SURMOF's rigid geometry fixes the separations of the linkers so that their separation is small enough to allow transport, but large enough to limit the intermolecular interactions that lead to aggregation. Further fine-tuning of these crystalline structures may result in strategies to engineer long excited-state transport lengths in specific crystallographic directions. Our initial analysis of singlet transport in the ADB layer suggests that significant singlet exciton transport lengths $(\sim 50 \mathrm{~nm})$ are reached in these crystalline thin films.

The C343@ADB SURMOFs are of general interest for applications requiring good PLQY. We investigated them for the specific application as an emitter layer for TTA-UC and found that hurdles remain to unlocking the potential of these emitter layers in upconversion. The emission spectrum of the C343@ADB SURMOF reverts to that of the monomer-like component of a pure ADB SURMOF upon excitation via triplet-triplet annihilation in a sensitizer/emitter SURMOF heterostructure. This suggests that transport of the triplets in the emitter away from the sensitizer/emitter interface is very limited. This allows for a very efficient back transfer of singlets generated by triplet-triplet annihilation in the emitter back into the sensitizer layer (especially from the singlets on 
the $\mathrm{C} 343$ acceptor). This back transfer mechanism counteracts the sought improvement of the UC PLQY in the ABD/C343 emitter layer. To overcome this hurdle, transport of triplets further into the emitter layer must be achieved, we are seeking to alter the orientation of the SURMOFs with respect to the heterojunction in order to favor transport in the direction perpendicular to the heterostructure interface. Favoring triplet transport towards and away from the interface should enhance the UC PLQY by decreasing the back-transfer with respect to the current situation wherein transport along the interface is favored. Although work remains to improve the UC PLQY to relevant levels, the potential of UC in dense ordered systems is confirmed in terms of access to low thresholds.

\section{Experimental Section}

\section{Synthesis of ADB SURMOF}

The 4,4'-(anthracene-9,10-diyl)dibenzoate (ADB) SURMOFs were prepared by following a liquid phase synthesis approach $^{49}$. Microscope cover slides were cleaned in an acetone ethanol $(1: 1 \mathrm{v} / \mathrm{v})$ solution. The surface was further cleaned using UV radiation and the hydrophilicity was increased by the creation of hydroxyl groups on top of the glass slide. Two solutions were prepared. One solution containing the organic linker $(20 \mu \mathrm{M})$ and the other solution the coordination metal $(0.2 \mathrm{mM})$. Both were sprayed cycle-wise on to the cover slips (15 seconds metal solution, 35 seconds waiting, 5 seconds rinsing with ethanol, 25 seconds linker solution, 35 seconds waiting, 5 seconds rinsing). For all samples 10 cycles were used. After preparation, the samples were immediately characterized and stored in the dark to minimize the effects of degradation.

\section{Synthesis of ADB/TPC SURMOF}

The SURMOFs with different ratios of $\mathrm{ADB}$ and $p$ terphenyl-4,4'-dicarboxylate (TPC) were prepared in a similar fashion to the ADB SURMOF. However, a certain amount of TPC was added to the ADB linker solution to give a desired molar ratio between $\mathrm{ADB}$ and TPC. We choose to use the molar ratios between ADB and TPC linker. For the 1:1 ratio the same amount of ADB and TPC solutions were mixed, both with a molar concentration of $20 \mu \mathrm{M}$. For the 10:1 ratio ten times the amount of TPC was added to an ADB solution. The samples were characterized immediately after preparation and stored in the dark to minimize effects of degradation.

\section{Synthesis of $C_{343} @ A D B$ and ADB/C 334 SURMOFs}

The infiltration of coumarin 343 (C343) and coumarin 334 (C334) was achieved by mixing the ADB solution together with the dye solution in fixed molar ratios (in mol. \%: 30, 20, $10,5,2,1,0.1,0.02,0.005)$. The mixture was sprayed as before. After preparation, the samples were immediately characterized and stored in the dark to minimize the effects of degradation.

\section{Synthesis of spin-coated SURMOFs}

The solution preparation was the same as for the spray coated SURMOFs. For Pd-DCP linker a concentration of 20 $\mu \mathrm{M}$ was used. The substrate was put onto the chuck of a spincoater and whilst spinning sequentially covered with the linker, an ethanol rinse, and then metal solution before repeating the process again from the linker step. For all steps the spinning speed was 2000 RPM.

\section{Infrared reflection-absorption spectroscopy}

The infrared reflection-absorption spectra were acquired with a resolution of $2 \mathrm{~cm}^{-1}$ using a FTIR spectrometer (Bruker VERTEX 80) under ambient conditions.

\section{$X$-Ray Diffraction (XRD):}

For the coplanar orientation, a Bruker D8 Advance instrument was used. The data were recorded over a $2 \theta$ range of $3.5-15^{\circ}$. The X-ray source was a $\mathrm{Cu}$-anode with $\mathrm{Cu} \mathrm{K} \mathrm{K}_{\alpha 1,2}$ radiation with a wavelength of $0.15419 \mathrm{~nm}$.

\section{Absorption measurement}

Absorption spectra were measured by using a PerkinElmer 950 spectrophotometer.

\section{PLQY and steady state emission measurement}

The PLQY measurements were performed according to de Mello et al..$^{33}$ The samples were excited inside an integrating sphere with $150 \mathrm{~mm}$ diameter using a $375 \mathrm{~nm}$ collimated beam from a LED. A fiber-coupled Avantes spectrometer was used to record the PL emission.

\section{Time resolved PL measurement}

A Hamamatsu Universal Streak Camera C10910 with Acton SpectraPro SP2300 spectrometer was employed for the time-resolved PL measurements. Lifetime measurements within a $12.5 \mathrm{~ns}$ window were performed using a Coherent Chameleon Compact OPO, which was pumped by a Chameleon femtosecond oscillator. The ADB SURMOF and the $\mathrm{ADB} / \mathrm{TPC}$ SURMOF were excited at $350 \mathrm{~nm}$. The spin-coated samples were excited at $380 \mathrm{~nm}$. All samples were maintained under dynamic vacuum at pressures $<10^{-4}$ mTorr to reduce the photo degradation due to oxygen exposure.

\section{Fluence dependent measurement}

For the fluence dependent PL measurements a femtosecond Ti-sapphire amplifier (Spectra-Physics Spitfire) was used with a pulse frequency of $3 \mathrm{kHz}$. The beam size was focused and measured to be $55 \mu \mathrm{m}$ in diameter. The emission was collected with a gated camera (Princeton Instruments iCCD Pimax3).

\section{Upconversion emission characterization}

The sample was excited with a $532 \mathrm{~nm}$ continuous wave laser and the emission spectrum was measured with a double monochromator (Bentham DTMS300) and a photomultiplier tube (Hamamatsu R928P). The emission was collimated into the monochromator by two 2" lenses. The emission was chopped after a short-pass filter with a fixed frequency and the signal was measured by a lock-in amplifier (Stanford SR830 DSP). The sample was held under dynamic vacuum at pressures $<10^{-4}$ mTorr to exclude oxygen quenching from altering the performance of the UC film.

\section{AUTHOR INFORMATION}

\section{Corresponding Author}

Ian A. Howard, ian.howard@kit.edu

\section{Present Addresses}

\section{Author Contributions}

MO synthesized the SURMOFs and made the measurements. DB and MJ contributed to the PLQY and time resolved measurements. IAH, AT, CW and BSR planned and guided the work. KC and JMH assisted with the exciton diffusion measurements and 
analysis. MO and IAH drafted the manuscript, which was corrected by all authors.

The authors declare no competing financial interests.

\section{ACKNOWLEDGMENT}

This work was supported by the Science and Technology of Nanosystems (STN) program of the Helmholtz Association and via the utilization of Karlsruhe Nano Micro Facility (KNMF). M.O. acknowledges support from Karlsruhe School of Optics and Photonics (KSOP) graduate school and for the research travel grant obtained from Karlsruhe House of Young Scientists (KHYS). I.A.H. thanks the BW Stiftung for financial support. M.O.S acknowledges grant support from the Science Foundation Ireland (IvP 13/IA/1894). B.S.R. would like to acknowledge the financial support provided by Helmholtz Recruitment Initiative Fellowship. 


\section{REFERENCES}

(1) Shekhah, O.; Liu, J.; Fischer, R. A.; Wöll, C. Chem. Soc. Rev. 2011, 40, 1081.

(2) Zhou, H.-C.; Long, J. R.; Yaghi, O. M. Chem. Rev. 2012, 112, 673 .

(3) Kent, C. A.; Mehl, B. P.; Ma, L.; Papanikolas, J. M.; Meyer, T. J.; Lin, W. J. Am. Chem. Soc. 2010, 132, 12767.

(4) Lee, C. Y.; Farha, O. K.; Hong, B. J.; Sarjeant, A. A.; Nguyen, S. T.; Hupp, J. T. J. Am. Chem. Soc. 2011, 133, 15858.

(5) Leong, K.; Foster, M. E.; Wong, B. M.; Spoerke, E. D.; Van Gough, D.; Deaton, J. C.; Allendorf, M. D. J. Mater. Chem. A 2014, 2, 3389.

(6) Lin, J.; Hu, X.; Zhang, P.; Van Rynbach, A.; Beratan, D. N.; Kent, C. A.; Mehl, B. P.; Papanikolas, J. M.; Meyer, T. J.; Lin, W.; Skourtis, S. S.; Constantinou, M. J. Phys. Chem. C 2013, 117, 22250

(7) So, M. C.; Wiederrecht, G. P.; Mondloch, J. E.; Hupp, J. T.; Farha, O. K. Chem. Commun. 2015, 51, 3501 .

(8) Son, H.-J.; Jin, S.; Patwardhan, S.; Wezenberg, S. J.; Jeong, N. C.; So, M.; Wilmer, C. E.; Sarjeant, A. A.; Schatz, G. C.; Snurr, R. Q.; Farha, O. K.; Wiederrecht, G. P.; Hupp, J. T. J. Am. Chem. Soc. 2013, 135,862 .

(9) Williams, D. E.; Rietman, J. A.; Maier, J. M.; Tan, R.; Greytak, A. B.; Smith, M. D.; Krause, J. A.; Shustova, N. B. J. Am. Chem. Soc. 2014, 136, 11886.

(10) Williams, D. E.; Shustova, N. B. Chem. Eur. J. 2015, 21, 15474 .

(11) Zhang, Q.; Zhang, C.; Cao, L.; Wang, Z.; An, B.; Lin, Z.; Huang, R.; Zhang, Z.; Wang, C.; Lin, W. J. Am. Chem. Soc. 2016, 138, 5308.

(12) Streit, H. C.; Adlung, M.; Shekhah, O.; Stammer, X.; Arslan, H. K.; Zybaylo, O.; Ladnorg, T.; Gliemann, H.; Franzreb, M.; Wöll, C.; Wickleder, C. ChemPhysChem 2012, 13, 2699.

(13) Kent, C. A.; Liu, D.; Ma, L.; Papanikolas, J. M.; Meyer, T. J.; Lin, W. J. Am. Chem. Soc. 2011, 133, 12940.

(14) Cao, L.; Lin, Z.; Shi, W.; Wang, Z.; Zhang, C.; Hu, X.; Wang, C.; Lin, W. J. Am. Chem. Soc. 2017, 139, 7020 .

(15) Stassen, I.; Burtch, N.; Talin, A.; Falcaro, P.; Allendorf, M.; Ameloot, R. Chem. Soc. Rev. 2017, 46, 3185 .

(16) Hendon, C. H.; Rieth, A. J.; Korzyński, M. D.; Dincă, M. ACS Cent. Sci. 2017, 3, 554.

(17) Lee, D. Y.; Shin, C. Y.; Yoon, S. J.; Lee, H. Y.; Lee, W.; Shrestha, N. K.; Lee, J. K.; Han, S.-H. Sci. Rep. 2014, 4, 3930.

(18) Liu, J.; Zhou, W.; Liu, J.; Howard, I.; Kilibarda, G.; Schlabach, S.; Coupry, D.; Addicoat, M.; Yoneda, S.; Tsutsui, Y.; Sakurai, T.; Seki, S.; Wang, Z.; Lindemann, P.; Redel, E.; Heine, T.; Wöll, C. Angew. Chem. Int. Ed. 2015, 54, 7441.

(19) Park, H. J.; So, M. C.; Gosztola, D.; Wiederrecht, G. P.; Emery, J. D.; Martinson, A. B.; Er, S.; Wilmer, C. E.; Vermeulen, N. A.; Aspuru-Guzik, A.;
Stoddart, J. F.; Farha, O. K.; Hupp, J. T. ACS Appl. Mater. Interfaces 2016, 8, 24983.

(20) Erickson, K. J.; Leonard, F.; Stavila, V.; Foster, M. E.; Spataru, C. D.; Jones, R. E.; Foley, B. M.; Hopkins, P. E.; Allendorf, M. D.; Talin, A. A. Adv. Mater. 2015, 27, 3453.

(21) Dou, J.-H.; Sun, L.; Ge, Y.; Li, W.; Hendon, C. H.; Li, J.; Gul, S.; Yano, J.; Stach, E. A.; Dincă, M. J. Am. Chem. Soc. 2017, 139, 13608.

(22) Talin, A. A.; Centrone, A.; Ford, A. C.; Foster, M. E.; Stavila, V.; Haney, P.; Kinney, R. A.; Szalai, V.; El Gabaly, F.; Yoon, H. P.; Léonard, F.; Allendorf, M. D. Science 2014, 343, 66.

(23) Wang, Z.; Liu, J.; Lukose, B.; Gu, Z.; Weidler, P. G.; Gliemann, H.; Heine, T.; Wöll, C. Nano Lett. 2014, 14, 1526.

(24) Shekhah, O.; Wang, H.; Kowarik, S.; Schreiber, F.; Paulus, M.; Tolan, M.; Sternemann, C.; Evers, F.; Zacher, D.; Fischer, R. A.; Wöll, C. J. Am. Chem. Soc. 2007, 129, 15118.

(25) Oldenburg, M.; Turshatov, A.; Busko, D.; Wollgarten, S.; Adams, M.; Baroni, N.; Welle, A.; Redel, E.; Wöll, C.; Richards, B. S.; Howard, I. A. Adv. Mater. 2016, $28,8477$.

(26) Haider, G.; Usman, M.; Chen, T. P.; Perumal, P.; Lu, K. L.; Chen, Y. F. ACS Nano 2016, 10 8366.

(27) Liu, J.; Lukose, B.; Shekhah, O.; Arslan, H. K.; Weidler, P.; Gliemann, H.; Brase, S.; Grosjean, S.; Godt, A.; Feng, X.; Mullen, K.; Magdau, I. B.; Heine, T.; Wöll, C. Sci. Rep. 2012, 2, 921.

(28) Arslan, H. K.; Shekhah, O.; Wohlgemuth, J.; Franzreb, M.; Fischer, R. A.; Wöll, C. Adv. Funct. Mater. 2011, 21, 4228 .

(29) Liu, J.; Wöll, C. Chem. Soc. Rev. 2017 46,5730 .

(30) Falcaro, P.; Okada, K.; Hara, T.; Ikigaki, K.; Tokudome, Y.; Thornton, A. W.; Hill, A. J.; Williams, T.; Doonan, C.; Takahashi, M. Nat. Mater. 2016, 16, 342.

(31) Dhakshinamoorthy, A.; Asiri, A. M.; Garcia, H. Catal. Sci. Technol. 2016, 6, 5238.

(32) de Juan, A.; Tauler, R. Crit. Rev. Anal. Chem. 2006, 36, 163.

(33) de Mello, J. C.; Wittmann, H. F.; Friend, R. H. Adv. Mater. 1997, 9, 230.

(34) Wilson, L. R.; Richards, B. S. Appl. Opt

2009, 48, 212

(35) Correa, N. M.; Levinger, N. E. J. Phys. Chem. B 2006, 110, 13050 .

(36) Reynolds G. A., D. K. H. Opt. Commun

$1975,13,222$

(37) Zaikowski, L.; Mauro, G.; Bird, M.; Karten, B.; Asaoka, S.; Wu, Q.; Cook, A. R.; Miller, J. R. J. Phys. Chem. B 2015, 119, 7231.

(38) Tamai, Y.; Matsuura, Y.; Ohkita, H.; Benten, H.; Ito, S. J. Phys. Chem. Lett. 2014, 5, 399.

(39) Mikhnenko, O. V.; Blom, P. W. M.; Nguyen, T.-Q. Energy Environ. Sci. 2015, 8, 1867. 
(40) Caram, J. R.; Doria, S.; Eisele, D. M.; Freyria, F. S.; Sinclair, T. S.; Rebentrost, P.; Lloyd, S.; Bawendi, M. G. Nano Lett. 2016, 16, 6808.

(41) Donati, D.; Williams, J. O. Mol. Cryst. Liq. Cryst. 1978, 44, 23.

(42) Tamai, Y.; Ohkita, H.; Benten, H.; Ito, S. J. Phys. Chem. Lett. 2015, 6, 3417.

(43) Hestand, N. J.; Spano, F. C. Acc. Chem. Res. 2017, 50, 341.

(44) Menke, S. M.; Luhman, W. A.; Holmes, R. J. Nat. Mater. 2013, 12, 152.

(45) Lunt, R. R.; Benziger, J. B.; Forrest, S. R. Adv. Mater. 2010, 22, 1233.
(46) Chernikova, V.; Shekhah, O.; Eddaoudi, M. ACS Appl. Mater. Interfaces 2016, 8, 20459.

(47) Monguzzi, A.; Tubino, R.; Hoseinkhani, S.; Campione, M.; Meinardi, F. Phys. Chem. Chem. Phys. 2012, 14, 4322 .

(48) Dilbeck, T.; Hill, S. P.; Hanson, K. J. Mater. Chem. A 2017, 5, 11652.

(49) Shekhah, O.; Wang, H.; Zacher, D.; Fischer, R. A.; Wöll, C. Angew. Chem. Int. Ed. 2009, 48, 5038 . 